\title{
Theorems in the Products of Related Quantities.
}

\author{
By F. H. JACrson, M.A.
}

$\S 1$. Let $(x)_{n}$ denote the function

then

$$
\underset{\kappa=\infty}{L} \frac{(x-n+1)(x-n+2) \cdots(x-n+\kappa)}{(x+1)(x+2) \cdots(x+\kappa)} \cdot \kappa^{n}
$$

$$
\begin{aligned}
& \frac{(x+r)_{n}}{|\underline{r}| \underline{0}}-\frac{(x+r-1)_{n}}{|\underline{\mid r-1}| \underline{1}}+\frac{(x+r-2)_{n}}{|\underline{r-2}| \underline{2}}- \\
& \cdots+(-1)^{r} \frac{(x)_{n}}{\underline{0} \mid \underline{r}}=\frac{n \cdot n-1 \cdot n-2 \cdots n-r+1}{\underline{r}}(x)_{n \rightarrow r} \cdots
\end{aligned}
$$

In Gamma Functions the above may be written.

$$
\begin{array}{r}
\frac{\Gamma(x)}{\Gamma(x-n)}-{ }_{r} C_{1} \frac{\Gamma(x-1)}{\Gamma(x-n-1)}+{ }_{r} C_{2} \frac{\Gamma(x-2)}{\Gamma(x-n-2)}-\ldots \ldots \\
\ldots+(-1)^{r} \frac{\Gamma(x-r)}{\Gamma(x-n-r)}=(n)_{r} \frac{\Gamma(x-r)}{\Gamma(x-n)} \ldots
\end{array}
$$

By using the theorem (1) I shall obtain a purely algebraical proof of the well-known theorem

$$
F_{1}(\alpha, \beta, \gamma)=\frac{\Pi(\gamma-1) \cdot \Pi(\gamma-\alpha-\beta-1)}{\Pi(\gamma-a-1) \Pi(\gamma-\beta-1)}
$$

where II denotes Gauss's II Function and $F_{1}(\alpha, \beta, \gamma)$ denotes the Hypergeometric Series in which the element $x_{x}=1$.

It can be deduced from (1) that

$$
\begin{aligned}
& 1-{ }_{r} \mathrm{C}_{1} \frac{(x-n)_{1}}{(x)_{1}}+{ }_{r} \mathrm{C}_{2} \frac{(x-n)_{2}}{(x)_{2}}-\cdots=\frac{(n)_{r}}{(x)_{r}} \text { a pretty analogy with...(3) } \\
& 1-{ }_{r} \mathrm{C}_{1} \frac{(x-n)^{1}}{x^{1}}+{ }_{r} \mathrm{C}_{2} \frac{(x-n)^{2}}{x^{2}}-\cdots=\frac{(n)^{r}}{(x)^{r}}
\end{aligned}
$$

$r$ is not necessarily an integer in (3) and (1).*

\section{* See $\$ 7$.}


\$2. $\Delta$ fundamental property of the function $(x)_{n}$ is

$$
(x)_{n} \times(x-n)_{m}=(x)_{n+m}
$$

whence we get $(x)_{n-r} \times(x-n+r)_{n} \times(x+r-s)_{r-s}=(x+r-s)_{n}$

Now the $(s+1)^{t h}$ term on the left side of $(1)=(-1)^{r} \frac{(x+r-s)_{n}}{\mid \underline{r-s \mid s}}$ which may be written

$$
(-1)^{s} \frac{(x)_{n-r}(x-n+r)_{s}(x+r-s)_{r-s}}{|\underline{r-s}| \underline{s}}
$$

Since $(x+r-s)_{r-s}$-when $r$ and $s$ are both integers-maybe, written in the form

$$
(x+1)(x+2)(x+3) \cdots(x+r-s)=(-1)^{r-s}(-x-1)_{r-s}
$$

$\therefore$ the $(s+1)^{t h}$ term $=(-1)^{r} \frac{(x)_{n \rightarrow r}(x-n+r)(-x-1)_{r-s}}{\underline{r-s} \mid s}$

The expression on the left side of (1) may be written

$$
\begin{aligned}
& (-1)^{r} \frac{(x)_{n-r}}{\mid \underline{r}}\left\{(-x-1)_{r}+\frac{\mid \underline{r}}{|\underline{r}-1| \underline{1}}(-x-1)_{r-1}(x-n+r)_{1}\right. \\
& +\frac{\mid \underline{r}}{\left.\underline{\underline{r-2} \mid \underline{2}}(-x-1)_{r-2}(x-u+r)_{2}+\cdots+(-1)^{r}(x-u+r)_{r}\right\}}
\end{aligned}
$$

By Vandermonde's theorem* the expression with the large bracket

$$
=(-x-1+x-n+r)=(r-n-1) \text {. }
$$

Expression (4) becomes

$$
(-1) \cdot \frac{(x)_{n-r}}{\underline{r}}(-n-1)_{r}=\frac{n \cdot n-1 \cdot n-2 \cdots n-r+1}{\underline{r}}(x)_{n-r}
$$

which proves theorem (1).

\footnotetext{
* Ste $\$ 7$.
} 
\$3. Now $\quad(x)_{n}=\frac{\Gamma(x+1)}{\Gamma(x-n+1)}$

$$
\because \Gamma(x+1)=\mathrm{L}_{\kappa=\infty} \frac{1 \cdot 2 \cdot 3 \cdot 4 \cdots \kappa}{(x+1)(x+2) \cdots(x+\kappa)} \kappa^{x}
$$

Replacing ( ) by Gamma Functions, the theorem (l), after multiplication throughout by $\mid \underline{r}$, becomes

$$
\begin{array}{r}
\frac{\Gamma(x+r+1)}{\Gamma(x+r-n+1)}-{ }_{r} \mathrm{C}_{1} \frac{\Gamma(x+r)}{\Gamma(x+r-n)}+{ }_{r} \mathrm{C}_{2} \frac{\Gamma(x+r-1)}{\Gamma(x+r-n-1)}-\cdots \\
\ldots+(-1)^{r} \frac{\Gamma(x+1)}{\Gamma(x-n+1)}=(n)_{r} \frac{\Gamma(x+1)}{\Gamma(x-n+r+1)}
\end{array}
$$

substitute $y$ for $x+r+1$, then (j) becomes

$$
\begin{array}{r}
\frac{\Gamma(y)}{\Gamma(y-n)}-{ }_{r} \mathrm{C}_{1} \frac{\Gamma(y-1)}{\Gamma(y-n-1)}+{ }_{r} \mathrm{C}_{2} \frac{\Gamma(y-2)}{\Gamma(y-n-2)}-\cdots \\
\ldots+(-1)^{r} \frac{\Gamma(y-r)}{\Gamma(y-n-r)}=(n)_{r} \frac{\Gamma(y-r)}{\Gamma(y-n)}
\end{array}
$$

Remembering that $\Gamma(y)=(y-1) \Gamma(y-1)$ on division throughout by $\frac{\Gamma(y)}{\Gamma(y-n)}$ we have $1-{ }_{r} \mathrm{C}_{1} \frac{(y-u-1)_{1}}{(y-1)_{1}}+{ }_{r} \mathrm{C}_{2} \frac{(y-u-1)_{2}}{(y-1)_{2}}-{ }_{r} \mathrm{C}_{3} \frac{(y-n-1)_{3}}{(y-1)_{3}}+\cdots=(n)_{r} \frac{1}{(y-1)_{r}}$ this may be written

$$
1-r \cdot \frac{(x-n)_{1}}{(x)_{1}}+\frac{r \cdot r-1}{2 !} \frac{(x-n)_{2}}{(x)_{2}}-\frac{r \cdot r-1 \cdot r-2}{3 !} \frac{(x-n)_{3}}{(x)_{3}}+\cdot \cdot=\frac{(n)_{r}}{(x)_{r}}
$$

analogous to the Binomial Expansion

$$
1-r \cdot \frac{(x-n)}{(x)}+\frac{r \cdot r-1(x-n)^{2}}{2 !}-\cdots=\frac{(n)^{r}}{(x)^{2}}
$$

The Expansion (5) has been obtained on the supposition that $r$ is a positive integer; but it will be shown later to hold for negative and fractional values of $r$. 
\$4. To consider the expansion in general of $f(x+y)$ in the form

$$
\mathrm{P}_{0}+\mathrm{P}_{1}(x)_{1}+\mathrm{P}_{2}(x)_{2}+\cdots+\mathrm{P}_{r}(x)_{r}+\cdots \cdot
$$

where $\mathbf{P}_{0} \cdot \mathbf{P}_{1} \cdot \mathbf{P}_{3} \cdots$ are functions of $y$ only or constants. Assume that $f(x+y)$ is capable of being expanded in a convergent series of the above form then

$$
f(x+y)=\mathbf{P}_{0}+\mathbf{P}_{1}(x)_{1}+\mathbf{P}_{2}(x)_{2}+\cdots+\mathbf{P}_{r}(x)_{r}+\cdots
$$

By giving $x$ the values $0 \cdot 1 \cdot 2 \cdot 3 \cdots \quad$ in succes sion we obtain the following equations to determine $\mathrm{P}_{0} \cdot \mathrm{P}_{1} \cdot \mathrm{P}_{2} \cdots$

$$
\begin{aligned}
f(y) \quad & =\mathrm{P}_{0} \\
f(y+1) & =\mathrm{P}_{0}+\mathrm{P}_{1} \\
f(y+2) & =\mathrm{P}_{0}+2 \mathrm{P}_{1}+2 \cdot 1 \mathrm{P}_{3} \\
& \cdots \cdots \cdots \cdots \cdots \\
f(y+r) & =\mathrm{P}_{0}+r \cdot \mathrm{P}_{1}+r \cdot r-1 \mathrm{P}_{2}+\ldots \mid \underline{r} \cdot \mathrm{P}
\end{aligned}
$$

From which we obtain

$$
\begin{aligned}
& \mathrm{P}_{0}=\frac{f(y)}{10} \\
& \mathrm{P}_{1}=\frac{f(y+1)}{\underline{0} \underline{0}}-\frac{f(y)}{\underline{0} \underline{1}}
\end{aligned}
$$

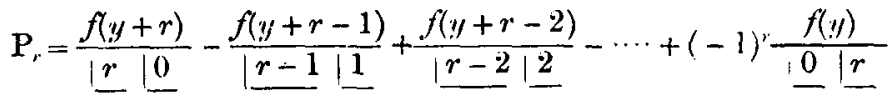

which is that

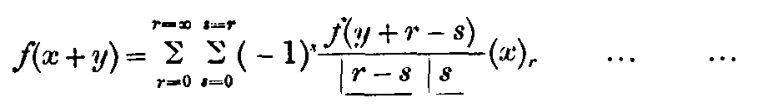

subject to the convergence of the series. 
§5. The expansion of $(x+y)_{n}, \quad n$ being unrestricted.

The coefficient of $x_{r}$ will be

$$
\begin{aligned}
& \mathrm{P}_{r} \equiv \frac{(y+r)_{n}}{\mid \underline{\underline{n}} \underline{0}}-\frac{(y+r-1)_{n}}{\frac{(y-1}{\mid \underline{1}}}+\frac{(y+r-2)_{n}}{\underline{|n-2| \underline{2}}}-\cdots \\
& \cdots+(-1)^{r} \frac{(y)_{n}}{10 \mid \underline{0}} \equiv \frac{n \cdot n-1 \cdots n-r+1}{\mid \underline{r}} y_{n \rightarrow r}
\end{aligned}
$$

(by Theorem (1)).

$$
\begin{aligned}
\therefore \quad(x+y)_{n}=y_{n}+n y_{n-1} x_{1} & +\frac{n \cdot n-1}{\frac{1}{2}} y_{n-2} x_{2}+\cdots \\
& +\frac{n \cdot n-1 \cdots x-r+1}{\underline{r}} y_{n-r} x_{r}+\cdots
\end{aligned}
$$

This is the generalised form of Vandermonde's Theorem; the proof depends, as will be seen on reference to $\$ 2$, No. 4, on Vandermonde's Theorem for positive integral values of the suffix.

To expand $a^{x}$ in a series of form (7)

we have $\mathrm{P}_{r}=\frac{a^{r}}{\underline{r} \underline{0}}-\frac{a^{r-1}}{\underline{r-1} L^{1}}+\cdots+(-1)^{r} \frac{a^{0}}{|\underline{0}| \underline{r}} \equiv \frac{(a-1)^{r}}{\mid \underline{r}}$

$\therefore a^{x}=1+(a-1)(x)_{1}+\frac{(a-1)^{2}}{\underline{L}}(x)_{2}+\cdots+\frac{(a-1)^{r}}{\underline{r}}(x)_{r}+\cdots$

this is a well known particular case of the Binomial Expansion.

To expand $\frac{1}{x+a}$

we have $P_{r}=\frac{1}{\underline{r}}\left\{\frac{1}{a+r}-{ }_{r} \mathrm{C}_{1} \frac{1}{a+r-1}+{ }_{r} \mathrm{C}_{2} \frac{1}{a+r-2}-\cdots+(-1)^{r}{ }_{a}^{1}\right\}$

$$
=\frac{1}{\mid r} \cdot \frac{\mid r}{(a+r)(a+r-1) \cdots(a+1) a} .
$$

$\therefore \quad \frac{1}{x+a}=\frac{1}{a}+\frac{(x)_{1}}{a \cdot a+1}+\frac{(x)_{2}}{a \cdot a+1 \cdot a+2}+\cdots \cdot+\frac{(x)_{r}}{a \cdot a+1 \cdots \cdot a+r}+\cdot \cdot$

This is a special case of Vandermonde's Theorem for negative integral values of the suffix. The functions which can be expanded in series of form (7) seem very restricted in number. 


\section{5}

\section{\$. Writing}

$$
\begin{gathered}
(x+y)_{n}=(y)_{n}+n \cdot(y)_{n-1}(x)_{1}+\frac{n \cdot n-1}{i \underline{\underline{2}}}(y)_{n-2}(x)_{2}+\cdots \\
\ldots+\frac{n \cdot n-1 \cdots n-r+1}{\mid \underline{r}}(y)_{n-r}(x)_{r}+\cdots
\end{gathered}
$$

divide both sides by $(y)_{n}$.

Then $\frac{(x+y)_{n}}{(y)_{n}}=1+n \cdot \frac{(y)_{n-1}(x)_{1}}{(y)_{n}}+\frac{n \cdot n-1}{\underline{12 ! r}} \frac{(y)_{n-2}(x)_{2}}{(y)_{n}}+\cdots$

Now it is easily seen that $\frac{(y)_{n-1}}{(y)_{n}}=\frac{1}{y-n+1}$

$$
\frac{(y)_{n-r}}{(y)_{n}}=\frac{1}{(y-n+1)_{r}}
$$

and $\frac{(x+y)_{n}}{(y)_{n}}=\frac{\Pi(x+y)}{\Pi(x+y-n)} \cdot \frac{\Pi(y-n)}{\Pi(x)}$ where $\Pi$ denotes Gauss's II Function. Therefore

$$
\begin{gathered}
\frac{\Pi(x+y) \cdot \Pi(y-n)}{\Pi(x+y-n) \Pi(x)}=1+n \cdot \frac{(x)_{1}}{(y-n+1)_{1}} \\
+\frac{n \cdot n-1}{2 !} \frac{(x)_{2}}{(y-n+1)(y-n+2)}+\cdots+\frac{n \cdot n-r+1}{r !} \frac{(x)_{r}}{(y-n+1)_{r}}+\cdots
\end{gathered}
$$

Replacing $n$ by $-\alpha, x$ by $-\beta$, and $y-n+1$ by $\gamma$ we have

$$
\begin{gathered}
\frac{\Pi(\gamma-a-\beta-1) \cdot \Pi(\gamma-1)}{\Pi(\gamma-\beta-1) \Pi(\gamma-a-1)}=1+\frac{\alpha \cdot \beta}{1 \cdot \gamma}+\frac{a \cdot \alpha+1 \cdot \beta \cdot \beta+1}{1 \cdot 2 \cdot \gamma \cdot \gamma+1}+\cdots \\
=\mathrm{F}_{1}(\alpha, \beta, \gamma)
\end{gathered}
$$

$\$ 7$. If in $\$ 2$, result (4), we had assumed the truth of Vandermonde's Theorem for unrestricted values of the suffix, Theorems (1), (2), and (3) would have been proved for all values of $r$. Since we have proved Vandermonde's Theorem for unrestricted values of the suffix, the proofs of $\$ 2$ and 3 may be repeated with $r$ unrestricted. The use of $(-1)^{r}$ in $\$ 2$ can easily be avoided. When $r$ is unrestricted, $\langle r\rangle_{r}$ must be used instead of $\underline{\mid r}$. 